\title{
Lymph Node Metastasis Predicts Disease Recurrence in a Single-Center Experience of 70 Stages 1 to 3 Appendix Cancers: A Retrospective Review
}

\author{
Garrett M. Nash, MD, MPH ${ }^{1}$, James D. Smith, MD¹, Laura Tang, MD², Martin R. Weiser, MD¹, \\ Larissa K. Temple, MD1, Eileen O'Reilly, MD³ , Leonard B. Saltz, MD³ , José G. Guillem, MD, \\ MPH $^{1}$, and Philip B. Paty, MD ${ }^{1}$ \\ ${ }^{1}$ Department of Surgery, Colorectal Service, Memorial Sloan-Kettering Cancer Center, New York, \\ NY, USA \\ ${ }^{2}$ Department of Pathology, Memorial Sloan-Kettering Cancer Center, New York, NY, USA \\ ${ }^{3}$ Department of Medicine, Memorial Sloan-Kettering Cancer Center, New York, NY, USA
}

\begin{abstract}
Background-Previous reports on the surgical management of appendix cancer show high recurrence rates among patients initially presenting with localized disease. This study sought to characterize predictors of outcome among patients treated for stages 1 to 3 appendix cancer at the authors' institution.
\end{abstract}

\begin{abstract}
Methods-Patients with nonmetastatic appendix cancer undergoing definitive surgery at a single cancer center from 1994 to 2013 were retrospectively reviewed. Patients with appendiceal adenomas, cystadenomas, or classical carcinoids were excluded from the study. The median follow-up period was 5.2 years (interquartile range, 2.9-6.7 years).
\end{abstract}

\begin{abstract}
Results-The study identified 70 patients, $49 \%$ of whom were women. The median age was 52 years (range, 20-84 years). All were explored by an expert surgeon who had treated at least 20 appendiceal cancers. The procedures were appendectomy $(n=2)$, right hemicolectomy $(n=66)$, and diagnostic laparoscopy and placement of an intraperitoneal port $(n=2)$. The final pathology showed that transmural (30 T4, $32 \mathrm{~T} 3,4 \mathrm{~T} 2,4 \mathrm{~T} 1)$ and node-negative disease (80 \%) was common. Goblet cell carcinoid (GCC) features were identified in $54 \%$ of the tumors. These were smaller and more likely to present as acute appendicitis than appendiceal adenocarcinoma (AA) but were otherwise similar in clinical presentation and outcome. The presence of lymph node (LN) metastasis was associated with a higher risk of recurrence than of stage 2 appendix cancer (78\% vs $4 \%$ at 5 years; $p<0.0001$ ). A total of 12 patients experienced recurrence ( 5 GCC, $7 \mathrm{AA}$ ): 9 in the peritoneum, 2 in mesenteric LNs, and 1 in the surgical incision.
\end{abstract}

\footnotetext{
Corresponding Author: Garrett M Nash, MD, MPH, FACS, Colorectal Service/Department of Surgery, Memorial Sloan-Kettering Cancer Center, 1275 York Avenue, Box 275, New York, NY 10065, USA, Phone: (646) 888-3086, ; Email: nashg@ mskcc.org Conflict of Interest There are no conflicts of interest.
} 
Conclusions-Stages 1 to 3 invasive AA and GCC behave similarly in terms of clinical presentation and outcome. Perforated appendix and T4 tumor stage were common but not associated with recurrence. Although uncommon, LN metastasis strongly predicted recurrence.

Three retrospective $<\mathrm{AQ} 1>$ series show generally poor outcomes of locoregional appendiceal adenocarcinoma (AA). The Massachusetts General Hospital (MGH) reports an overall recurrence rate of $60 \%$ for stages 1 to 3 disease. The Mayo Clinic reports $33 \%$ recurrence for stage 2 and $50 \%$ for stage 3 disease, and the German multicenter series reports $0 \%$ to $12 \%$ recurrence for stage $1,21 \%$ to $41 \%$ for stage 2 , and $61 \%$ to $75 \%$ for stage 3 disease. ${ }^{1-3}$

The Surveillance, Epidemiology, and End Results (SEER) program and other data sources suggest that the incidence of appendiceal cancer is approximately 2.6 per 1 million people per year. ${ }^{4-6}$ However, the histologic diagnosis of noncarcinoid tumors in the appendix has been confusing and may have led to inaccurate recording of the true incidence. Furthermore, tumors of the appendix containing neuroendocrine and glandular differentiation have commonly been described as adenocarcinoid or goblet cell carcinoid (GCC) and classified by some as a variety of classical carcinoid. However, a review of 63 such cases by Tang et al. ${ }^{7}$ demonstrated that the behavior of GCC is akin to that of invasive adenocarcinoma of the appendix.

Unfortunately, a majority of all AAs, including GCCs, present with diffuse peritoneal disease rather than as a localized tumor found incidentally at the time of appendicitis, unrelated surgery, or imaging studies. ${ }^{8}$ Given the limited literature on primary appendix cancer, we reviewed the Memorial Sloan Kettering Cancer Center (MSK) experience with locoregional AA and GCC, aiming to describe the frequency and pattern of recurrence and to find indicators of prognosis that may guide selection of surgical and adjuvant therapy.

\section{Methods}

Data were extracted from a prospective database and from the electronic medical records of patients treated at a single cancer center from January 1994 to April 2013. All the patients who had undergone laparotomy or laparoscopy at MSK for invasive AA or GCC were included in the study. All the surgeons had experience with at least 20 cases of appendiceal cancer. A single pathologist (L.T.) reviewed the available surgical slides to confirm the diagnosis of either AA or GCC. ${ }^{7}$ If slides were not available for a second review, pathology data were abstracted from the original review performed by specialized gastrointestinal cancer pathologists at MSK. The exclusion criteria specified the absence of invasive cancer or the presence of classical carcinoid or metastatic disease.

Of the 70 patients in the study, $<\mathrm{AQ} 2>57$ had undergone an appendectomy, whereas 2 had undergone a right colectomy at an outside hospital and were referred to our institution for additional therapy. For these patients, tumor size was abstracted from the outside pathology reports. All 59 patients subsequently underwent laparotomy or laparoscopy at MSK. The remaining 11 patients had primary surgery at MSK. Patients with a prior diagnosis of appendix cancer were staged preoperatively (before appendectomy or right colectomy) with computed tomography (CT) scanning of the abdomen and pelvis. 
Patients were selected for adjuvant chemotherapy by a multidisciplinary team of surgeons and oncologists and received systemic 5-fluorouracil (5-FU) with or without oxaliplatin or irinotecan and/or early postoperative intraperitoneal chemotherapy (EPIC) with floxuridine (FUDR). Therapeutic decisions were based on data extrapolated from studies showing a benefit of 5-FU-based therapy for stage 3 and, to a lesser extent, stage 2 colon cancer. Between 1994 and 2000, 5-FU/leucovorin was typically used. Combination therapy with irinotecan (IFL) became available after 2000, and combination therapy with oxaliplatin (FOLFOX) became available after 2003.

Patients were selected to receive one to six cycles of EPIC (median, 3 cycles) based on colon cancer risk stratification (e.g., T4, perforated tumor) and patient preference. The duration of EPIC therapy varied based on the treating oncologist and the patient's ability to tolerate treatment. Neoadjuvant therapy was not used.

The median patient follow-up period was 5.2 years (interquartile range, 2.9-6.7 years). Time to recurrence was determined from the electronic medical records of all the study patients, including the surgeon and oncologist office notes as well as the radiology, operative, and pathology reports. Pathologic confirmation of recurrence was available for 10 patients. The remaining 2 patients $<\mathrm{AQ} 3>$ had clinical and radiologic progression and died of the recurrence.

The patients were followed clinically. Typically, they underwent a history, physical exam, and carcinoembryonic antigen (CEA) test every 3 to 6 months, and serial CT scans at least once a year. The date of the first radiologic evidence of recurrence and the site of recurrence were recorded. Time to recurrence (i.e., interval from date of diagnosis to date of first radiologic evidence of recurrence) was estimated using Kaplan-Meier analysis. Patients were censored for recurrence at the time of their last follow-up visit.

Subgroups were compared by the log-rank test, and statistical significance was defined as a $p$ value lower than 0.05 . Proportions of categorical variables were compared using the chisquare test unless expected cell counts were lower than 5 (in which case, Fisher's exact test was used). Statistical analyses were conducted using SPSS version 17.0 (SPSS, Chicago, IL, USA).

\section{Results}

The study identified 70 patients with stages 1 to 3 AA or GCC. Age and stage at presentation were similar for the two histologic subtypes (Table 1). $<\mathrm{T} 1>$ Among AA tumors, 5 were well differentiated, 22 were moderately differentiated, and 2 were poorly differentiated. Grade was not documented for three patients. According to the Tang classification of GCC, 28 were type A (typical GCC), 6 were type B (signet ring), 4 were type $\mathrm{C}$ (poorly differentiated). The patients with GCC were more likely to present with symptomatic appendicitis than the patients with AA ( $87 \%$ vs $53 \% ; p=0.002)$. The AA tumors were larger than the GCC tumors (mean size, $4.1 \mathrm{vs} 2.4 \mathrm{~cm} ; p=0.04$ ). Similar proportions of patients had a clinically or pathologically perforated appendix or an appendix removed in fragments. 
At our institution, 66 patients subsequently underwent right colectomy, 2 underwent diagnostic laparoscopy and placement of an intraperitoneal port (after right colectomy at an outside hospital), and 2 underwent appendectomy alone. The median number of lymph nodes retrieved in the colon resection patients was 21 (range, 3-44). Adjuvant chemotherapy was used for 35 patients, whereas 5 patients received EPIC alone, 15 received systemic chemotherapy alone, and 15 received both EPIC and systemic chemotherapy.

Use of adjuvant therapy did not vary based on histology. Approximately half of the patients with stage 2 cancer and almost all those with stage 3 cancer received some form of adjuvant chemotherapy (Table 2). $<\mathrm{T} 2>$ Two stage 3 patients did not receive adjuvant therapy. The one patient refused it, and the remaining patient was not fit for chemotherapy due to postoperative complications.

Of the 68 patients who underwent right colectomy, 12 subsequently had recurrent disease, whereas neither of the appendectomy-alone patients experienced recurrence. Of the 12 patients who experienced recurrence, 9 had peritoneal-only recurrence, 2 had mesenteric lymph node recurrence, and 1 had an incisional recurrence. The 5-year estimated recurrence rate was $19 \%$, with no recurrence identified after 4 years of follow-up evaluation.

In the univariable analysis, the only identified patient or tumor factor associated with recurrence was the presence of lymph node metastasis (Table 3). $<\mathrm{T} 3>$ Age, gender, appendicitis at diagnosis, appendix or tumor perforation, histologic type, tumor size, and tumor stage were not associated with recurrence. Multivariable analysis was not possible due to the small study cohort $(n=70)$ and the infrequency of tumor recurrence $(n=12)$.

\section{Discussion}

In this series of patients with locoregional appendiceal cancer, we found that recurrence (19\% at 5 years) was much lower and disease-specific survival (95\% at 5 years) much higher than we had anticipated based on the existing literature (Table 4). $<\mathrm{T} 4\rangle$ The only predictor of recurrence was the presence of lymph node metastasis ( $78 \%$ vs $4 \%$ ).

Interestingly, this study had no examples of isolated solid organ recurrence. Acute appendicitis was not associated with recurrence. Whereas the MGH report suggested that perforation may be a risk factor for recurrence, ${ }^{2}$ this was not supported by the Mayo series ${ }^{1}$ or by our data. Overall, we found that $50 \%$ of the patients had perforated appendix due to tumor perforation, suppurative appendicitis, or fragmentation during appendectomy. This was common with or without recurrence (67\% vs $48 \%$, respectively; $p=0.34$ ). Our dataset may have been too small to detect variables exerting a small effect on oncologic outcomes. However, we do not recommend that perforation be considered a risk factor in the absence of supportive evidence.

The average size of recurring tumors was not greater than that of nonrecurring tumors. However, no way exists to validate these measurements, which may be difficult to perform accurately due to inflammatory reaction at the time of appendectomy. As a result, we do not think it appropriate to use tumor size to prognosticate or guide treatment. Additionally, we found that AA and GCC have a similar pattern of presentation and clinical course. This 
finding is similar to that of a previous MSK study predominantly including patients with metastatic appendix cancer. In the metastatic setting, type A had the best prognosis and type $\mathrm{C}$ the worst. However, in stages 1 to 3 GCC patients, recurrences were among only types A and B. Thus, type of GCC cannot be used to make management decisions in this clinical context. Both AA and GCC behave quite differently from the more indolent classical carcinoid (CC). We therefore believe it is not useful to group GCC with CC, as was done in the German study.

Compared with the published literature, our key finding was an association of good oncologic outcome with node-negative cancer despite the fact that the majority of such patients had a T3 or T4 tumor or perforated appendix. Possible explanations are numerous and difficult to determine conclusively. However, several are immediately apparent: possible referral bias, small sample size in each of the published reports, confusing and inconsistently applied pathologic classification systems throughout the literature, combined analysis of patients with and without metastatic disease in the prior studies, absence of universally accepted surgery and chemotherapy protocols, and incomplete patient follow-up evaluation.

We have addressed some limitations of prior studies by applying a consistent pathology classification system, staging all patients with surgery and cross-sectional imaging, and establishing thorough follow-up evaluation. We previously attempted to refine the pathologic classification system to identify patients with similar cancer biology. Expert pathologic review of available samples was provided by a gastrointestinal specialized pathologist with extensive appendix cancer experience. However, although all patients had a staging or therapeutic operation at our institution, 59 underwent appendectomy or right colectomy at another hospital before definitive surgery at MSK, and all sections of each tumor specimen may not have been available for secondary review. To avoid including patients with unrecognized metastatic disease, we included only those without metastasis shown by imaging or surgical exploration. The previously published series do not describe staging procedures. The worse outcomes in other series may reflect understaging due to missed peritoneal disease.

We cannot definitively conclude that right colectomy confers therapeutic benefit for patients with appendiceal cancer because our study had no control group. However, surgical exploration and right colectomy allow staging of lymph nodes and the peritoneum. This is invaluable in prognosis and selection for adjuvant therapy. Furthermore, if we are to extrapolate from the colon cancer treatment surgical paradigm — and we have no basis to argue against that-right colectomy appears reasonable because it is the only way to clear locoregional disease. In $26 \%$ of our patients (15/57), residual cancer was identified in the colon wall or lymph nodes in the right colectomy specimen.

Some important limitations persist. Our center treats a disproportionate number of younger appendix cancer patients (median age, 52 years) seeking aggressive management, and as a referral center, we may not capture the full spectrum of this uncommon disease. Use of chemotherapy for stage 2 disease in this series was heterogeneous and varied based on individual patient, surgeon, and oncologist preferences. Therefore, it is impossible to determine the potential incremental benefit of adjuvant intraperitoneal or systemic 
chemotherapy for patients with locoregional appendix cancer. Unfortunately, given the rarity of the disease, it is impractical to expect an adequately powered randomized trial to measure any benefit of adjuvant chemotherapy. However, given the $96 \% 5$-year recurrence-free survival rate in this cohort, it is unlikely that adjuvant therapy would have had much impact on stage 2 tumors. Therefore, we doubt that the heterogeneity of chemotherapeutic approaches had a significant impact on the reported outcomes. For optimal oncologic outcome to be achieved and future comparative studies to be conducted, we recommend that the appendix cancer specimen be reviewed by an expert pathologist and that plans for subsequent adjuvant chemotherapy be carefully considered before definitive surgical treatment. Given the apparently low risk of recurrence in the absence of lymph node metastasis, we consider that routine use of adjuvant therapy after right hemicolectomy for all patients with stage 2 appendix cancer is not justified. However, placement of an intraperitoneal port at the time of right colectomy may be considered in the event that lymph node metastasis is identified in the surgical specimen or in stage 2 patients whose pathology (based on colon cancer data) suggests a higher risk of recurrence. This would make it possible to deliver EPIC in addition to systemic chemotherapy to appendix cancer patients at extremely high risk for peritoneal-based recurrence.

\section{Acknowledgments}

This study was funded in part by the cancer center Core Grant P30 CA008748. The Core Grant provides funding to institutional cores such as biostatistics and pathology, which were used in this study.

\section{References}

1. Nitecki SS, Wolff BG, Schlinkert R, Sarr MG. The natural history of surgically treated primary adenocarcinoma of the appendix. Ann Surg. 1994; 219:51-7. [PubMed: 8297177]

2. Proulx GM, Willett CG, Daley W, Shellito PC. Appendiceal carcinoma: patterns of failure following surgery and implications for adjuvant therapy. J Surg Oncol. 1997; 66:51-3. [PubMed: 9290693]

3. Benedix F, Reimer A, Gastinger I, Mroczkowski P, Lippert H, Kube R. Study Group Colon/Rectum Carcinoma Primary Tumor. Primary appendiceal carcinoma-epidemiology, surgery and survival: results of a German multi-center study. Eur J Surg Oncol. 2010; 36:763-1. AQ4. [PubMed: 20561765]

4. McGory ML, Maggard MA, Kang H, O'Connell JB, Ko CY. Malignancies of the appendix: beyond case series reports. Dis Colon Rectum. 2005; 48:2264-71. [PubMed: 16258711]

5. Connor SJ, Hanna GB, Frizelle FA. Appendiceal tumors: retrospective clinicopathologic analysis of appendiceal tumors from 7,970 appendectomies. Dis Colon Rectum. 1998; 41:75-80. [PubMed: 9510314]

6. O'Donnell ME, Badger SA, Beattie GC, Carson J, Garstin WI. Malignant neoplasms of the appendix. Int J Colorectal Dis. 2007; 22:1239-48. [PubMed: 17447078]

7. Tang LH, Shia J, Soslow RA, et al. Pathologic classification and clinical behavior of the spectrum of goblet cell carcinoid tumors of the appendix. Am J Surg Pathol. 2008; 32:1429-43. [PubMed: 18685490]

8. Sugarbaker PH. The natural history, gross pathology, and histopathology of appendiceal epithelial neoplasms. Eur J Surg Oncol. 2006; 32:644-7. [PubMed: 16621426] 


\section{Synopsis}

Previous reports show high recurrence rates among patients initially presenting with localized appendix cancer. This study sought to characterize predictors of outcome for stages 1 to 3 appendix cancer. Perforated appendix and T4 tumor stage were not associated with recurrence. Lymph node metastasis strongly predicted recurrence. 


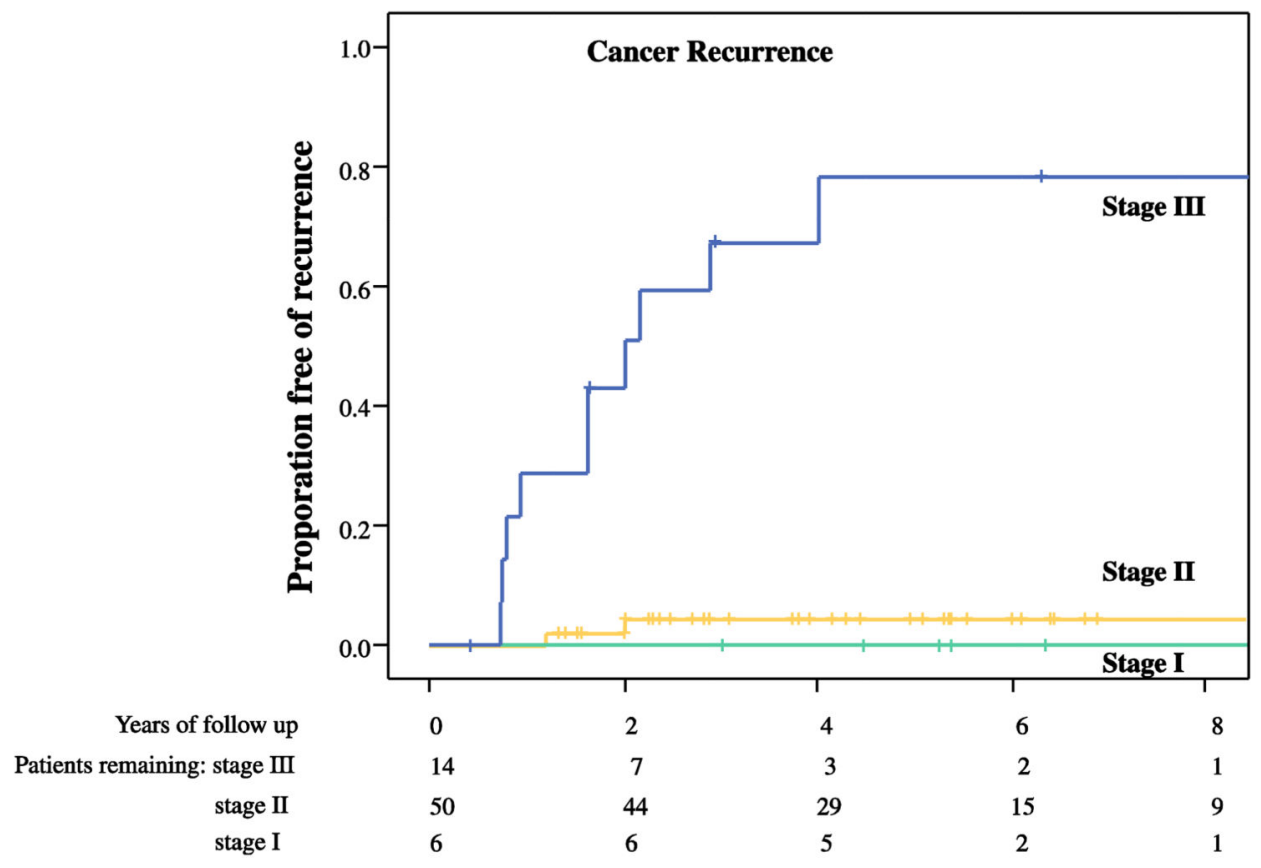

Fig. 1.

Cancer recurrence. 


\section{Table 1}

Initial presentation

\begin{tabular}{|c|c|c|c|}
\hline & Adenocarcinoma & Goblet cell carcinoid & $p$ Value \\
\hline$n$ & 32 & 38 & \\
\hline Female $(\%)$ & 50 & 47 & 0.99 \\
\hline Mean age (years) & $53 \pm 15$ & $54 \pm 14$ & 0.56 \\
\hline Mean tumor size $(\mathrm{cm})$ & $4.1 \pm 2.7$ & $2.4 \pm 1.6$ & 0.04 \\
\hline Acute appendicitis (\%) & 53 & 87 & 0.002 \\
\hline Perforated appendix $(\%)^{a}$ & 59 & 46 & 0.23 \\
\hline Stage $1(\%)$ & 9 & 8 & 0.27 \\
\hline Stage $2(\%)$ & 63 & 79 & \\
\hline Stage $3(\%)$ & 28 & 13 & \\
\hline
\end{tabular}

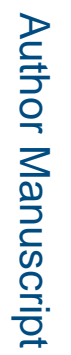

${ }^{a}$ Clinical or pathologic perforation or appendix removed in fragments 
Table 2

Use of adjuvant systemic and/or intraperitoneal chemotherapy

\begin{tabular}{lll}
\hline & Adenocarcinoma $\boldsymbol{n}(\boldsymbol{\%})$ & Goblet cell carcinoid $\boldsymbol{n}(\boldsymbol{\%})$ \\
$n(\%)$ & $18 / 32(56)$ & $17 / 38(45)$ \\
Stage 1 & $0 / 3(0)$ & $0 / 3(0)$ \\
Stage 2 & $10 / 20(50)$ & $13 / 30(43)$ \\
Stage 3 & $8 / 9(89)$ & $4 / 5(80)$ \\
\hline
\end{tabular}


Table 3

Comparison of patients with and without recurrence

\begin{tabular}{llll}
\hline & Recurrence $(\boldsymbol{n}=\mathbf{1 2})$ & No recurrence $(\boldsymbol{n}=\mathbf{5 8})$ & $\boldsymbol{p}$ Value \\
Female gender $(\%)$ & 33 & 52 & 0.25 \\
Mean age (years) & $53 \pm 12$ & $54 \pm 15$ & 0.94 \\
Acute appendicitis (\%) & 92 & 67 & 0.16 \\
Perforated appendix (\%) & 67 & 48 & 0.34 \\
Goblet cell carcinoid (\%) & 42 & 57 & 0.36 \\
Mean tumor size (cm) & $4.1 \pm 1.9$ & $3.2 \pm 2.5$ & 0.25 \\
T4 (\%) & 50 & 41 & 0.75 \\
Node positive (\%) & 83 & 7 & $<0.0001$ \\
Adjuvant therapy $(\%)$ & 92 & 41 & 0.003 \\
\hline
\end{tabular}


Table 4

\section{Comparison of outcomes between published series of appendix cancer}

\begin{tabular}{lllll}
\hline & Series $(n)$ & Stage 1\%(n) & Stage 2 \% (n) & Stage 3\%(n) \\
5-Year recurrence & $\operatorname{MSKCC}(70)$ & $0 \%(6)$ & $4(50)$ & $78(14)$ \\
& $\operatorname{MGH}^{2}(15)$ & $60(15)^{a}$ & & \\
5-Year disease-specific survival & $\operatorname{MSKCC~(70)~} 100(6)$ & $97(50)$ & $83(14)$ \\
& $\operatorname{Mayo}^{1}(67)$ & $100(9)$ & $67(37)$ & $50(21)$ \\
& $\operatorname{German}^{3}$ (NA) & $100(\mathrm{NA})$ & $79-88(\mathrm{NA})$ & $25-39(\mathrm{NA})$ \\
\hline
\end{tabular}

MSKCC, Memorial Sloan Kettering Cancer Center; MGH, Massachusetts General Hospital; NA, number of patients not available by stage

${ }^{a}$ Recurrence not reported by stage 